\title{
Superior Frontal Gyrus
}

National Cancer Institute

\section{Source}

National Cancer Institute. Superior Frontal Gyrus. NCI Thesaurus. Code C33674.

A large region of the frontal lobe of the brain located above the superior frontal sulcus that participates with the primary motor cortex in the control and initiation of voluntary movements and possibly contributes to higher cognitive functions. 